\title{
Balantidiasis: reporte de cuatro casos y revisión de la casuística de los hospitales de Lima.
}

\author{
Balantidiasis: Report of four cases.
}

\author{
PAMO Oscar ${ }^{1}$, FIGUEROA Marcos ${ }^{2}$, RUIZ Jorge ${ }^{2}$ \\ ${ }^{1}$ Profesor Asociado, Departamento de Medicina-UPCH. \\ ${ }^{2}$ Bachiller en Medicina-UPCH.
}

La balantidiasis humana tiene un comportamiento clínico muy semejante al de la amebiasis. En nuestro medio, J,M. Fernández la describió en 1901 (1). Desde entonces se han publicado trabajos esporádicos y aislados con excepción de los realizados por H.Lumbreras (2). Diversos estudios de campo llevados a cabo entre 1942 y 1962 mostraron una prevalencia de 0 a $10 \%$ de portadores asintomáticos en diversas zonas del país. En los cerdos, animales que son considerados como parte de la cadena biológica, las prevalencias han sido del 50 al $70 \%(3,4)$.

Los casos de balantidiasis humana que suelen verse en los hospitales son de formas disentéricas crónicas. Entre 1985 y 1990 fueron hospitalizados cuatro pacientes con ese diagnóstico en el Hospital Dos de Mayo en Lima.

\section{Caso $\mathbf{N}^{\circ} 1$}

H.C \# 889304. Varón de 68 años, natural y procedente de Canta (Lima), agricultor, que ingresó el 17-10-86 con una historia de diarrea sin moco ni sangre de 7 meses de evolución. Los exámenes auxiliares mostraron un hematocrito de $36 \%$, hemograma normal, albúmina plasmática de $2.04 \mathrm{gr} \%$ y un estudio radiológico que reveló un probable cáncer gástrico. En el examen de heces se halló trofozoítos de B. coli. El paciente fue tratado con tetraciclinas por vía oral, cesando la diarrea; sin embargo, el estado general se deterioró. El paciente optó por el retiro voluntario ante la posibilidad quirúrgica, desconociéndose el curso ulterior.

\section{CASO $\mathrm{N}^{\circ} 2$}

H.C. \# 971287. Varón de 68 años, natural y procedente de Moyapata (Cuzco), agricultor, ingresó el 05-03-88, con un tiempo de enfermedad de cuatro meses y una historia de diarrea disentérica, dolor abdominal, disuria y hematuria. En el examen físico se constató un abdomen con dolor difuso pero sin signos peritoneales y el tacto rectal reveló una tumoración ulcerada en la ampolla rectal. El Hcto fue de $32 \%$ con una cuenta normal de leucocitos con desviación izquierda, albúmina plasmática de $2.16 \mathrm{gr} \%$ y leucocituria más hematuria en el sedimento urinario. En la sigmoidoscopía se halló una tumoración irregular, a $10 \mathrm{~cm}$. del margen anal, ulcerada, cubierta de exudado serofibrinoso. Se tomaron biopsias con la presunción de carcinoma rectal. La diarrea con sangre y el dolor abdominal continuaron hasta que el paciente hizo rectorragia severa con choque hipovolémico que lo llevó a la muerte. El estudio anatomo- 
patológico mostró inflamación aguda en la mucosa rectal, presencia de B. coli en las zonas necróticas y en la lámina propia de la mucosa y ausencia de tejido neoplásico.

\section{CASO $\mathbf{N}^{\circ} 3$}

H.C. \# 967614. Varón de 87 años, natural y procedente de San Rafael (Huanuco), agricultor, ingresó el 04-04-88 con dos semanas de enfermedad, caracterizada por diarrea sin moco ni sangre pero con tenesmo, epigastralgia y vómitos postprandiales. En el examen clínico lucía crónicamente enfermo, emaciado y con dolorabilidad epigástrica a la palpación. Tenía un Hcto de 41 y 8600 leucocitos, con una albuminemia de 3.3gr\%. El paciente se agravó rápidamente e hizo retención nitrogenada. El examen de heces reveló la presencia de trofozoitos de B. coli. No obstante que se inició el tratamiento con sulfato de aminosidina más cloramfenicol, el paciente falleció con perforación intestinal y sepsis.

\section{CASO $\mathrm{N}^{\circ} 4$}

H.C.\# 1026129. Varón de 65 años, natural y procedente de Cajamarca, agricultor, ingresó el 12-02-90 con un tiempo de enfermedad de tres semanas, con una historia de diarreas con moco, sangre y tenesmo, dolor cólico en mesogastrio e hipogastrio y náuseas y vómitos postprandiales. Al examen se halló: deshidratado, leve hepatomegalia y agrandamiento prostático. El Hcto. Fue de 39 con 7300 leucocitos y albuminemia de $2.64 \mathrm{gr} \%$. En el examen de heces se encontró huevos de T. trichiura y trofozoitos de B. coli. El paciente fue tratado con doxiciclina y albendazol, y se recuperó gradualmente para ser dado de alta.

\section{DISCUSIÓN}

Los casos 3 y 4 estuvieron bajo nuestra atención y tuvimos conocimiento cercano de lo sucedido con el caso 2. Todo ello nos motivó a revisar la estadística del hospital y constatamos que sólo había hospitalizado un paciente, (caso 1), con el diagnóstico de balantidiasis, en los últimos 5 años. Esta búsqueda se extendió a otros hospitales generales de Lima, dependencias del Ministerio de Salud, como el Hospital Arzobispo Loayza (de mujeres), el Hospital Cayetano Heredia y el Hospital Hipólito Unanue, encontrándose 23 pacientes hospitalizados con este diagnóstico (Ver cuadro $\mathrm{N}^{\circ} 1$ ).

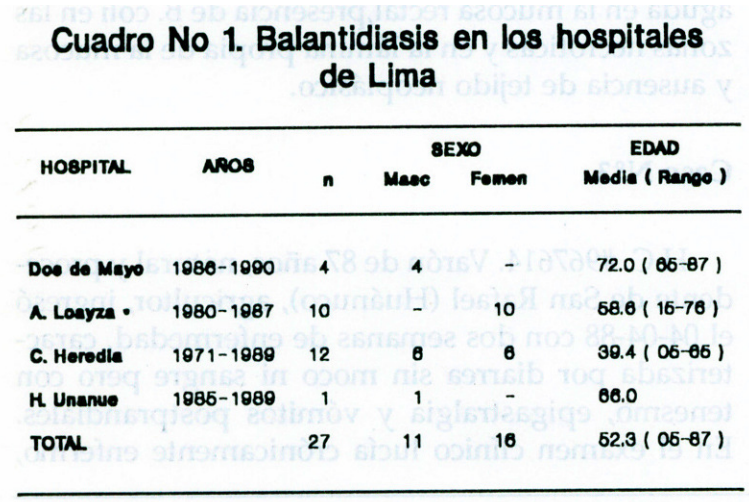

- No Incluye a 2 casos oltados en la referencla blbllograflce (18) 
Once $(40.7 \%)$ fueron varones y $16(59.30 \%$ mujeres. Ocho $(30.7 \%)$ procedían de las provincias de Lima, 7 (26.9\%) de Ancash, 3 (11.5\%) de Junín, 2 (7.7\%) de la Libertad 2 (7.7\%) de Cajamarca y 1 de Cuzco, Ayacucho, Arequipa y Apurímac, respectivamente. La mayoría fue analfabeta (55.6\%) y dedicados a la agricultura $(59.2 \%)$.

Considerando a tres pacientes que refirieron un tiempo de enfermedad cerca de un año, el promedio fue 142.7 días (rango de 6 a 912 días). Las diarreas disentéricas estuvieron presentes en 25 de ellos $(92.6 \%)$ y el antecedente de la crianza de cerdos sólo fue referido por 8 pacientes (29.6\%). De los exámenes auxiliares, la hipoalbuminemia fue una constante, promedio de $2.83 \mathrm{gr} / \mathrm{dl}$. El diagnóstico se hizo por la presencia de trofozoítos en el examen de heces en 26 de ellos (96\%) y por el examen anatomopatológico en uno. El diagnóstico fue tardío por lo general; siendo el promedio de 11.2 días (rango de 1 a 63 días) en 18 pacientes.

El tiempo de hospitalización fue prolongado, con un promedio de 30.2 días (rango de 6 a 115 días). Los dos pacientes (7.4\%) que fallecieron presentaron hemorragia digestiva baja severa, uno de ellos con choque hipovolémico y el otro con perforación intestinal y sepsis. La coexistencia de otros procesos patológicos dificultó las apreciaciones iniciales: 2 con infección urinaria, 1 con tuberculosis ganglionar, 2 con cáncer gástrico, 3 con insuficiencia renal, 1 con síndrome nefrótico, 1 con absceso perinéfrico, 1 con hipoplasia medular y 8 con otras parasitosis intestinales ( 2 con trichuriasis, 2 con estrongiloidiasis, 2 con uncinariasis, 1 con ascariasis y otro con isosporidiasis). Dieciséis $(51.6 \%)$ recibieron sulfato de aminosidina y $12(38.7 \%)$ fueron tratados con tetraciclinas.

El Batantidium coli es el protozoario más grande (alcanza hasta 200 micras) y único ciliado de importancia médica que parasita a la especie humana. Tiene una distribución cosmopolita y rara vez produce enfermedad en el ser humano. Así que hasta 1960 se había descrito 722 casos de balantidiasis.

El B.coli parasita primariamente el intestino grueso del humano. Por lo menos se conoce otros 33 huéspedes animales, de especies del género Balantidium y especies indistinguibles del B.coli, entre los que se encuentran los cerdos, ratas, monos y cobayos (5).

Las formas quísticas que se eliminan en las heces son las formas infectantes y pueden resistir varias semanas en un medio húmedo. Una vez ingerido los quistes, estos resisten la acidez gástrica y en el medio alcalino o neutro del intestino delgado se desenquistan para dar lugar a los trofozoítos que van a alojarse en el colon produciendo un proceso inflamatorio y necrosis tisular en el huésped susceptible. La gran mayoría son asintomáticos (6), unos presentan diarreas de evolución crónica y/o recurrente, mientras que otros hacen las formas disentéricas agudas. Los dos primeros cuadros son propios de las zonas endémicas. También se ha descrito complicaciones como perforación intestinal y hemorragia digestiva severa, abscesos hepáticos, compromiso pulmonar y cardíaco, etc. que suelen ser mortales $(7,8,9,10)$.

La epidemiología de la balantidiasis es compleja. Al parecer el cerdo no es el único ni el más importante factor en la transmisión. Por ejemplo, se ha demostrado la ocurrencia de la enfermedad entre enfermos mentales y entre los musulmanes que no tienen contacto con cerdos. Del mismo modo, no existe una mayor ocurrencia entre el personal que 
labora con cerdos. Mientras que ha sido posible transmitir la enfermedad del hombre a los animales, los intentos para infectar a humanos voluntarios han sido fallidos. Mucho depende del estado nutricional del huésped, de su capacidad inmunológica, de la coexistencia de otras parasitosis y/o infecciones intestinales, etc. y de otras patologías concurrentes $(11,12,13)$.

Los casos clínicos reportados son hechos aislados pero se ha descrito verdaderos brotes epidémicos entre enfermos mentales o relacionados a catástrofes como el tifón de la Isla de Truk (14).

Para el diagnóstico es necesario que el examen directo se realice en las heces recién emitidas o frescas y con solución salina. En el Perú, la "técnica de Baermann modificada en copa" ha dado buenos resultados $(15,3)$.

En el tratamiento se puede usar las tetraciclinas; por ejemplo, la oxitetraciclina a razón de $500 \mathrm{mg} . q 6 \mathrm{~h}$. por 10 días o la doxiciclina, $100 \mathrm{mh} . q 12 \mathrm{~h}$. por 10 días. También existen los tratamientos cortos con el sulfato de paramomicina y el sulfato se aminosidina, 500 mg.q6h. hasta completar 5 dosis $(2.5 \mathrm{~g})$ para ambos $(16,17)$. Aunque ha sido descontinuada, la diiodohidroxiquina, a dosis de $650 \mathrm{mg}$. tres veces al día por 20 días, puede ser una alternativa. El metronidazol es inefectivo pero otro imidazólico, el nimorazol ha dado muy buenos resultados a la dosis de $25 \mathrm{mg}$. por peso diario por cinco días. El tratamiento debe individualizarse, sobretodo si existe otros procesos patológicos. Muchas veces se deberá usar la vía parenteral, cobertura antibiótica amplia para los casos complicados y otras medidas de soporte en general.

\section{BIBLIOGRAFÍA}

1.Fernández, J.M. La disentería balantidiana. Tesis de Bachiller. Universidad Mayor de San Marcos Lima-Perú 1901.

2.Lumbreras, H. Balantidiasis en el Perú. Distribución geográfica, clínica, anatomía patológica y terapéutica. Revista Médica Peruana 1954; 25(311): 463-531.

3.Lumbreras H, y Muñoz N. El uso de la "técnica de Baermann modificada en copa" para determinar la incidencia de la Balantidiasis en la ciudad de Pucallpa del departamento de Loreto, Perú. Revista Médica Peruana 1962, 31 (331): 83-88.

4.Santibañez J, Vásquez V, Muñoz $\mathrm{P}$ y Cárdenas Z. Estudio epidemiológico y coproparasitario de grupos familiares con casos índices de Balantidiasis humana en Chile. Parasitol Dia 1985, 9(2):51-54.

5.Collet JY, Galdikas BM, Sugarjito J y Jojosudharmo SA. Coprological study of parasitism in orangutans in Indonesia. J Med Primatol 1986; 15(2): 121-9.

6.Vásquez V, Muñoz P, y Santibañez J. Balantidiasis humana en las Islas de Chiloe (al sur de Chile). Parasitol. Dia. 1987, 11 (4): 51-54.

7.Daoudal P, Wagschal G, Delacour JL et al. Balantidiose pulmonaire. Un cas en Franche-Comte. Presse Med 1986, 15(6) :257.

8.Ladas SD, Savva S, Frydas A et al. Invasive balantidiasis presented as chronic colitis and lung involvement. Dig Dis Sci 1989; 34 (10):1621-3.

9.Vidan JR, Frauca A, Martínez B y Borda F. Parasitosis hepática por Balantidium coli. Med clin (Barcelona). 1985, 85(7): 299-300

10.Zárate CE, Sánchez H, Rodríguez HD, Valdivia RM y Nava CE. Perforación intestinal por Balantium coli. Presentación de dos casos. Rev Gastroent Perú 1986; 6(1): $55-55$ 
11.Kamberoglou D, Savva S, Adraskelas N, et al. Balantidiasis complicating a case of ulcerativecolitis. Am J Gastroenterol 1990; 85(6): 765-6

12. Moraleda L, Franjola R, Zamorano P, y Hott H. Tricuriasis masiva asociada a balantidiasis y a trichostrongilosis. Rev Med Chile 1985, 113(10):1004-6.

13.Quiroga ML y Cornejo BG. Colitis pseudomembranosa y balantidiasis: informe de un caso. Salud Volví 1985, 3(1):48-52.

14. Walzer PD, Judson FN, Murphy KB, et al. Balantidiasis outbreak in Truk. The Am J. Of Trop Med and Hyg 1973; 22(1): 33-41

15.Cantella R. Y Burga Acha R. Balantidiasis y Strongyloidosis en el valle de Chanchamayo. Revista Médica Peruana 1963, XXXII (333):49-58.

16.Lumbreras H. Tratamiento de la Balantidiasis humana con dosis bajas de sulfato de paromomicina. Informe de un nuevo caso. Revista Médica Peruana 1963; 32(33):65-67. 17.Lumbreras H, León R, Cantella R, Burga R. Y Reyes T. Tratamiento de la Balantidiasis con el nuevo antibiótico sulfato de aminosidina. Revista Médica Peruana 1963, 32 (333):59-63. 